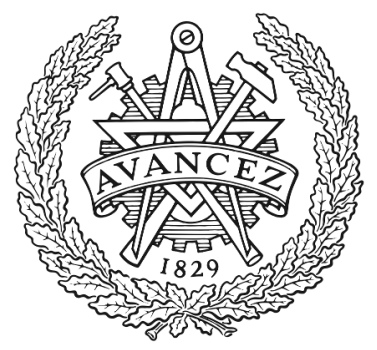

CHALMERS

UNIVERSITY OF TECHNOLOGY

\title{
Indoor Mapping with a Mobile Radar Using an EK-PHD Filter
}

Downloaded from: https://research.chalmers.se, 2023-04-26 14:02 UTC

Citation for the original published paper (version of record):

Talvitie, J., Kaltiokallio, O., Rastorgueva-Foi, E. et al (2021). Indoor Mapping with a Mobile Radar Using an EK-PHD Filter. IEEE International Symposium on Personal, Indoor and Mobile Radio Communications, PIMRC, 2021-September. http://dx.doi.org/10.1109/PIMRC50174.2021.9569630

N.B. When citing this work, cite the original published paper. 


\title{
Indoor Mapping with a Mobile Radar Using an EK-PHD Filter
}

\author{
Jukka Talvitie $^{\dagger}$, Ossi Kaltiokallio ${ }^{\dagger}$, Elizaveta Rastorgueva-Foi ${ }^{\dagger}$, Carlos Baquero Barneto ${ }^{\dagger}$, \\ Musa Furkan Keskin ${ }^{\star}$, Henk Wymeersch ${ }^{\star}$, and Mikko Valkama ${ }^{\dagger}$ \\ ${ }^{\dagger}$ Unit of Electrical Engineering, Tampere University, Tampere, Finland \\ ${ }^{\star}$ Department of Electrical Engineering, Chalmers University of Technology, Göteborg, Sweden
}

\begin{abstract}
Integrated communications, localization and sensing is one of the most addressed technologies considered for future mobile communications systems. In this context, a user equipment (UE)-centric mobile radar has been proposed to introduce improved situational awareness, and consequently potential improvement in network performance. In this paper, we derive an extended Kalman probability hypothesis density (EK-PHD) filter with a novel feature model, for a mobile radar based environment mapping, where range-angle detections are used to track map objects over time for dynamic map construction. In order to evaluate the performance of the proposed filtering approach, we employ a realistic ray-tracing-based simulation setup, which models the full transmission chain from the transmitted IQsamples to mapping results. Besides this, a simplified measurement model considering solely single-bounce specular reflections is exploited for providing further insight into the filter performance. The obtained results show that the proposed EK-PHD filter is able to provide high-quality mapping results, reaching around $10 \mathrm{~cm}$ landmark estimation accuracy in the considered millimeter wave simulation setup.
\end{abstract}

Index Terms - mobile radar, environmental mapping, millimeter wave, probability hypothesis density, extended Kalman filter

\section{INTRODUCTION}

The development of the current $5 \mathrm{G}$ and upcoming $6 \mathrm{G}$ mobile radio technologies is turning a conventional user equipment (UE) into a multi-functional device capable of performing integrated communications, localization and sensing via common radio frequency (RF) resources [1]. The currently available millimeter wave (mmWave) bands in $5 \mathrm{G}$ new radio (NR), which provide signal bandwidths of hundreds of megahertz, are being extended towards the terahertz frequency range offering several gigahertz of contiguous bandwidth. From the localization and sensing perspective, the higher carrier frequencies and larger bandwidths anticipate improved performance via increased resolution for ranging and angular estimation.

One of the emerging use cases for future mobile networks is the environment sensing and mapping with a joint radar and communications design [2]-[8]. Within this scope, a UE-centric mobile radar is studied in [5]-[8], where the fundamental idea is to utilize signals of the underlying communications system for enabling the sensing functionality. In [5] a channel

Corresponding author: Jukka Talvitie (jukka.talvitie@tuni.fi). This workwas partially supported by the Academy of Finland (grants \#319994, \#323244, \#328214, \#338224), Business Finland under the project 5G VIIMA, Tampere University Graduate School, and MSCA-IF grant 888913 (OTFS-RADCOM). model is proposed for personal radars by conducting a channel measurement campaign with massive arrays at the mmWave frequency range. In [7] and [8] indoor mapping is considered according to a static sensing approach, where measurements from different time instants are processed independently. In both studies, an empirical measurement campaign is applied to justify the presented mapping results, including also a specific focus on mapping with 5G NR signals in [8]. In [6], an extended Kalman filter (EKF) is utilized for dynamic mapping based on a grid-based approach, where the area of interest is divided into small cells known a priori by the radar. Besides mapping results, the study provides a trade-off analysis considering aspects on angular resolution, channel scanning time, used signal bandwidth and ranging accuracy. Complementary to these works on pure mapping, in [9]-[12] different approaches are considered in the context of simultaneous localization and mapping (SLAM), where the UE position information is estimated by means of an auxiliary anchor node or provided as a priori in the beginning of the mapping process. In [12], the utilization of a probability hypothesis density (PHD) filter is discussed in the context of SLAM with comprehensible illustrations and analysis of the filter functioning. Moreover, in [10], a PHD filter was introduced to a SLAM setting by mapping multipath components of a downlink radio link into a dynamically updated radio map. A PHD filter has several desirable properties, such as built-in data association between the radio map and obtained radio sensing measurements, and its ability of tracking different types of landmarks in the radio map, such as walls and scatterer objects.

There are several challenges with existing approaches for mobile radar mapping: grid-based mapping, e.g. [6], requires a lot of memory, and the mapping accuracy is subject to the chosen grid granularity. When approaching to cm-level sensing accuracy, such as in 6G networks, these can be considered as major challenges for the grid-based approach. Due to this, using a feature-based mapping, such as via PHD filtering, is preferred for the considered framework. However, the featurebased formulation of the PHD filter used in a SLAM setting, such as in [10], is not directly applicable to the UE-centric mobile radar scenario, because the modeling of walls by fixed virtual anchor is violated due to UE mobility.

In this paper, we propose using an extended Kalman proba- 
bility hypothesis density (EK-PHD) filter for dynamic environment mapping with a UE-centric mobile radar by considering standardized RF signals from the underlying communications system. We address the aforementioned challenges by a new model for large reflective surfaces. The main contributions of this paper are summarized as follows:

- We derive an efficient EK-PHD filter for the considered UE-centric dynamic mapping scenario, consisting of multiple objects and rich multipath propagation environment.

- We propose a new feature model for mapping large objects, arising naturally from mapping with mobile radars.

- We utilize a realistic indoor ray-tracing setup for EKPHD performance evaluation, considering the full radio transmission pipeline from the transmitted IQ-samples to range-angle measurements.

- To obtain quantitative performance results with standard metrics and gain deeper insight, we evaluate and analyze a simplified simulation setup, where radar measurements are obtained from predefined statistical distributions.

\section{System Model and Range-Angle Measurements}

\section{A. System Model}

For the considered mobile radar concept, we utilize a multiple-input multiple-output (MIMO) channel model based on co-located transmit and receive antenna arrays equipped with analog RF beamforming capability, and $N_{\mathrm{T}}$ and $N_{\mathrm{R}}$ antenna elements, respectively. For the purpose of radio sensing and mapping, the UE transmits a sequence of $M$ orthogonal frequency-division multiplexing (OFDM) symbols with $N$ active subcarriers. Assuming appropriate synchronization and cyclic prefix $(\mathrm{CP})$ removal, the received sample at the $m^{\text {th }}$ OFDM symbol and $n^{\text {th }}$ subcarrier is defined as [13]

$$
y_{m, n}=\mathbf{f}_{\mathrm{R}}^{H} \mathbf{A}_{\mathrm{R}, n} \boldsymbol{\Gamma}_{n} \mathbf{A}_{\mathrm{T}, n}^{H} \mathbf{f}_{\mathrm{T}} x_{m, n}+n_{m, n},
$$

where $\mathbf{f}_{\mathrm{T}} \in \mathbb{C}^{N_{\mathrm{T}} \times 1}$ and $\mathbf{f}_{\mathrm{R}} \in \mathbb{C}^{N_{\mathrm{R}} \times 1}$ are the analog transmitter and receiver beamformers, $x_{m, n}$ is the transmitted symbol, and $n_{m, n}$ is complex Gaussian noise. Moreover, multipath propagation over $L$ radio paths is captured by the path coefficient matrix

$$
\begin{gathered}
\boldsymbol{\Gamma}_{n}=\operatorname{diag}\left(\gamma_{1, n}, \gamma_{2, n}, \ldots, \gamma_{L, n}\right) \in \mathbb{C}^{L \times L} \\
\text { with } \gamma_{l, n}=b_{l} e^{-j 2 \pi n \Delta f \tau_{l}},
\end{gathered}
$$

and the steering matrices

$$
\begin{aligned}
& \mathbf{A}_{\mathrm{T}, n}=\left[\mathbf{a}_{\mathrm{T}, n}\left(\phi_{\mathrm{T}}^{1}\right), \mathbf{a}_{\mathrm{T}, n}\left(\phi_{\mathrm{T}}^{2}\right), \ldots, \mathbf{a}_{\mathrm{T}, n}\left(\phi_{\mathrm{T}}^{L}\right)\right] \in \mathbb{C}^{N_{\mathrm{T}} \times L}, \\
& \mathbf{A}_{\mathrm{R}, n}=\left[\mathbf{a}_{\mathrm{R}, n}\left(\phi_{\mathrm{R}}^{1}\right), \mathbf{a}_{\mathrm{R}, n}\left(\phi_{\mathrm{R}}^{2}\right), \ldots, \mathbf{a}_{\mathrm{R}, n}\left(\phi_{\mathrm{R}}^{L}\right)\right] \in \mathbb{C}^{N_{\mathrm{R}} \times L},
\end{aligned}
$$

where $\Delta f$ is the OFDM subcarrier spacing, $b_{l}$ is the complex channel coefficient, and $\tau_{l}$ is the propagation delay for the $l^{\text {th }}$ path. In addition, $\phi_{\mathrm{T}}^{l}=\left[\theta_{\mathrm{T}}^{l}, \psi_{\mathrm{T}}^{l}\right]$ and $\phi_{\mathrm{R}}^{l}=\left[\theta_{\mathrm{R}}^{l}, \psi_{\mathrm{R}}^{l}\right]$ are the departure and arrival angles of the $l^{\text {th }}$ path, respectively, where $\theta_{\mathrm{T}}^{l}\left(\right.$ and $\theta_{\mathrm{R}}^{l}$ ) denote the azimuth angle and $\psi_{\mathrm{T}}^{l}\left(\right.$ and $\psi_{\mathrm{R}}^{l}$ ) the elevation angle. Furthermore, $\mathbf{a}_{\mathrm{T}, n}\left(\phi_{\mathrm{T}}^{l}\right)$ and $\mathbf{a}_{\mathrm{R}, n}\left(\phi_{\mathrm{R}}^{l}\right)$ are steering vectors for the transmitter and receiver array in respective order, whose formulation is dependent on the considered array element geometry. In case that the ratio between the transmission bandwidth and carrier frequency is very small, the steering vectors follow a narrow-band model with fixed response over all subcarriers $n$.

\section{B. Range-Angle Estimation}

For the proposed mapping approach, discussed in Section III, the UE obtains range-angle measurements of the tracked targets, namely the incident points of scattering or specular reflections for each path. In this paper, we consider mapping in $2 \mathrm{D}$, which is sufficient for estimating, for example, a building floor plan. At the $k^{\text {th }}$ measurement instant, the range-angle measurements of possible targets are obtained in the form of $z_{k}^{j}=\left[d_{k}^{j} \theta_{k}^{j}\right]^{\top}$, where $d_{k}^{j}$ is the estimated distance between the $\mathrm{UE}$ and the target, and $\theta_{k}^{j}$ is the corresponding azimuth angle of the target.

For acquiring the range-angle measurements, we utilize the approach described in [14], where an efficient $\ell_{1}$-regularized least squares is used to provide sparse range-angle measurements via an analog beamsweeping procedure. With respect to this, assuming co-located transmitter and receiver antenna arrays as well as a single bounce radio path, the range measurement $d_{k}^{j}$ can be obtained via estimating the propagation delay $\tau_{l}$ of the corresponding radio path in (1)-(2) by $d_{k}^{j} \triangleq \tau_{l} c / 2$, where $c$ is the speed of light. Moreover, following the same assumptions, the target angle $\theta_{k}^{j}$ is equal to the departure and arrival angles of the corresponding radio path in (3) as $\theta_{k}^{j} \triangleq \theta_{\mathrm{T}}^{l}=\theta_{\mathrm{R}}^{l}$. The $2 \times 2$ measurements covariance matrix is denoted by $\Sigma$.

\section{Proposed Mapping Approach}

A multiple-target system can be characterized by modeling the multiple-target set $\mathcal{X}_{k}=\left\{x^{1}, \ldots, x^{n_{k}}\right\}$, with $x \in \mathbb{X}$ and measurement set $\mathcal{Z}_{k}=\left\{z_{k}^{1}, \ldots, z_{k}^{m_{k}}\right\}$ as random finite sets (RFSs) which capture the collection of single target states $x^{i}$ and measurements $z_{k}^{j}$. This paper aims to recursively estimate first-order statistical moment of the multiple-target posterior density, $p\left(\mathcal{X}_{k} \mid \mathcal{Z}_{1: k}\right)$, i.e., its PHD $v(x) .{ }^{1}$ The local maxima of $v(x)$ are points in $\mathbb{X}$ and can be used to generate estimates for the elements of $\mathcal{X}$.

\section{A. Basics of the PHD Filter}

The PHD can be propagated in time through the following recursion [16]

$$
\begin{aligned}
v_{k \mid k-1}(x)= & P^{\mathrm{S}} \int f_{k \mid k-1}(x, \zeta) v_{k-1}(\zeta) \mathrm{d} \zeta+v_{k}^{\mathrm{B}}(x), \\
v_{k}(x)= & \left(1-P^{\mathrm{D}}\right) v_{k \mid k-1}(x) \\
& +\sum_{z \in \mathcal{Z}_{k}} \frac{P^{\mathrm{D}} h_{k}(z \mid x) v_{k \mid k-1}(x)}{\lambda_{c}+P^{\mathrm{D}} \int h_{k}(z \mid \xi) v_{k \mid k-1}(\xi) \mathrm{d} \xi},
\end{aligned}
$$

where $P^{\mathrm{S}} \in[0,1]$ and $P^{\mathrm{D}} \in[0,1]$ denote the survival and detection probabilities in respective order, $f(\cdot)$ the transition

\footnotetext{
${ }^{1}$ For $\mathcal{X}$ with elements in state space $\mathbb{X}$ with probability distribution $p$, its first-order moment is a non-negative function $v$ on $\mathbb{X}$, such that for each region $S \subseteq \mathbb{X}[15] \int|\mathcal{X} \cap S| p(\mathrm{~d} \mathcal{X})=\int_{S} v(x) \mathrm{d} x$. where $|\mathcal{X}|$ denotes the cardinality of a set $\mathcal{X}$. The integral gives the expected number of elements in $\mathcal{X}$ that are in $S$.
} 


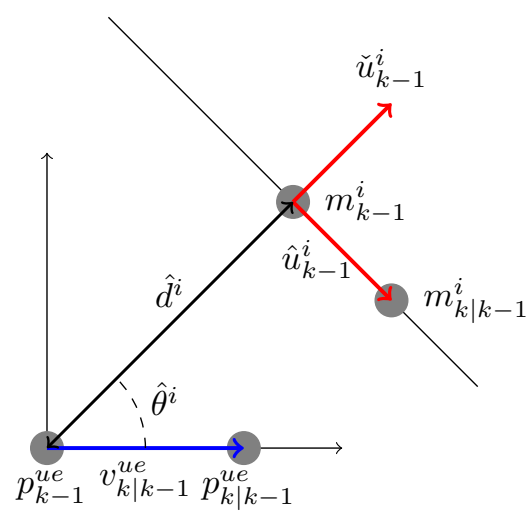

Fig. 1. Problem geometry, where known UE movement leads to a deterministic change of the estimated incidence point along the surface.

density, $h(\cdot)$ the likelihood function, $v_{k}^{\mathrm{B}}(\cdot)$ PHD of the birth RFS, $\lambda_{c}$ the clutter intensity, and $\zeta$ and $\xi$ denote the previous state. Modeling the PHD as a Gaussian mixture (GM),

$$
v_{k}(x)=\sum_{i=1}^{J_{k}} w_{k}^{i} \mathcal{N}\left(x ; m_{k}^{i}, P_{k}^{i}\right),
$$

the PHD recursion in (4)-(5) admits a closed-form solution [15]. Parameters of the GM in (6) are the number of components $J$, the component weight $w$, and the component mean $m$ and covariance $P$. In this paper, the integrals in the PHD recursion are solved by locally linearizing the nonlinear models and the prediction and update steps of the developed EK-PHD filter are presented in the following.

\section{B. Prediction Step}

Assuming that the posterior PHD at time $k-1$ is a GM of the form given in (6), it follows that the predicted PHD is also a GM defined as [15]:

$$
v_{k \mid k-1}(x)=P^{\mathrm{S}} \sum_{i=1}^{J_{k \mid k-1}} w_{k \mid k-1}^{i} \mathcal{N}\left(x ; m_{k \mid k-1}^{i}, P_{k \mid k-1}^{i}\right)+v_{k}^{\mathrm{B}}(x),
$$

where the first term represents the surviving objects and the latter term is the birth process capturing all appearing landmarks. For scattering, the incidence point remains stationary and parameters of the predicted density are: $w_{k \mid k-1}^{i}=w_{k-1}^{i}$, $m_{k \mid k-1}^{i}=m_{k-1}^{i}, P_{k \mid k-1}^{i}=P_{k-1}^{i}$. For specular reflection on the other hand, the incidence point changes as a function of UE location and this change must be taken into account as illustrated in Fig. 1. The surface can be parameterized using a line and parameters of the predicted density are given by

$$
\begin{aligned}
w_{k \mid k-1}^{i} & =w_{k-1}^{i}, \\
m_{k \mid k-1}^{i} & =m_{k-1}^{i}+t_{k \mid k-1}^{i} \hat{u}_{k-1}^{i}, \\
P_{k \mid k-1}^{i} & =F_{x}\left(m_{k-1}^{i}\right) P_{k-1}^{i}\left[F_{x}\left(m_{k-1}^{i}\right)\right]^{\top},
\end{aligned}
$$

where $t_{k \mid k-1}^{i}=\left(v_{k \mid k-1}^{u e}\right)^{\top} \hat{u}_{k-1}^{i}$ defines the scalar vector projection of $v_{k \mid k-1}^{u e}$ onto $\hat{u}_{k-1}^{i}$. The vector, $v_{k \mid k-1}^{u e}$, is known and it captures the UE movement, whereas the unit vector, $\hat{u}_{k-1}^{i}$, defines the surface orientation and it can be estimated using $\hat{u}_{k-1}^{i}=A^{i} \check{u}_{k-1}^{i}$, where $A^{i}$ is a $90^{\circ}$ rotation matrix, $\check{u}_{k-1}^{i}=\left[\cos \left(\hat{\theta}^{i}\right) \sin \left(\hat{\theta}^{i}\right)\right]^{\top}$ and $\hat{\theta}^{i}=\arctan \left(\frac{\hat{y}-y^{u e}}{\hat{x}-x^{u e}}\right)$. The Jacobian of the transition density is

$$
\begin{aligned}
& F_{x}\left(m_{k-1}^{i}\right)=t_{k \mid k-1}^{i} A^{i} \partial \hat{u}^{i}+I_{2}, \\
& \partial \hat{u}^{i}=\left[\begin{array}{cc}
\frac{a^{2}}{\left(\hat{x}-x^{u e}\right)\left(1+a^{2}\right)^{3 / 2}} & \frac{y^{u e}-\hat{y}}{\left(\hat{x}-x^{u e}\right)^{2}\left(1+a^{2}\right)^{3 / 2}} \\
\frac{\left(y^{u e}-\hat{y}\right)\left(1+a^{2}\right)+a^{3}\left(\hat{x}-x^{u e}\right)}{\left(\hat{x}-x^{u e}\right)^{2}\left(1+a^{2}\right)^{3 / 2}} & \frac{\left(\hat{x}-x^{u e}\right)^{2}\left(1+a^{2}\right)-\left(\hat{y}-y^{u e}\right)^{2}}{\left(\hat{x}-x^{u e}\right)^{3}\left(1+a^{2}\right)^{3 / 2}}
\end{array}\right],
\end{aligned}
$$

in which the indices $i$ and $k \mid k-1$ are omitted for brevity, $\left[\begin{array}{ll}x^{u e} & y^{u e}\end{array}\right]$ denotes the UE coordinates, $\left[\begin{array}{ll}\hat{x} & \hat{y}\end{array}\right]^{\top} \triangleq m_{k-1}^{i}, a=$ $\left(\hat{y}-y^{u e}\right) /\left(\hat{x}-x^{u e}\right)$ and $I_{2}$ is the $2 \times 2$ identity matrix.

The birth PHD is also represented as a GM and the parameters are: $w_{k}^{\mathrm{B}, j}, m_{k}^{\mathrm{B}, j}$ and $P_{k}^{\mathrm{B}, j}$. For each measurement that is not associated to an existing landmark ${ }^{2}$ during the previous update step, a birth is generated for both landmark types since the source of the measurement is unknown. As an example, birth $j$ is generated utilizing measurement $z_{k}^{j}=\left[\begin{array}{ll}d_{k}^{j} & \theta_{k}^{j}\end{array}\right]^{\top}$, and the mean and covariance are given by

$$
\begin{aligned}
m_{k}^{\mathrm{B}, j} & =\left[\begin{array}{ll}
x_{k}^{u e} & y_{k}^{u e}
\end{array}\right]^{\top}+d_{k}^{j} \cdot\left[\begin{array}{ll}
\cos \left(\theta_{k}^{j}\right) & \sin \left(\theta_{k}^{j}\right)
\end{array}\right]^{\top}, \\
P_{k}^{\mathrm{B}, j} & =\left(\left[\begin{array}{ll}
{\left[H_{x}\left(m_{k}^{\mathrm{B}, j}\right)\right.}
\end{array}\right]^{\top} \Sigma^{-1} H_{x}\left(m_{k}^{\mathrm{B}, j}\right)\right)^{-1},
\end{aligned}
$$

where $H_{x}\left(m_{k}^{j}\right)$ is the Jacobian defined in Section III-C.

\section{Update Step}

Under the assumptions that the landmarks generate observations independently and that clutter and predicted RFS are Poisson, it can be shown that the posterior of the PHD can be updated using [15]

$$
v_{k \mid k}(x)=\left(1-P^{\mathrm{D}}\right) v_{k \mid k-1}(x)+\sum_{z \in \mathcal{Z}_{k}} v_{k \mid k}^{\mathrm{D}}(x ; z),
$$

where the first term represents objects that are undetected and the latter term depicts the set of detected objects. Components of the detected objects are given by [15]

$$
\begin{aligned}
v_{k \mid k}^{\mathrm{D}}(x ; z) & =\sum_{i=1}^{J_{k \mid k-1}} w_{k \mid k}^{i}(z) \mathcal{N}\left(x ; m_{k \mid k}^{i}, P_{k \mid k}^{i}\right), \quad \text { where } \\
w_{k \mid k}^{i}(z) & =\frac{P^{\mathrm{D}} w_{k \mid k-1}^{i} \mathcal{N}\left(z ; h\left(m_{k \mid k-1}^{i}\right), S_{k}^{i}\right)}{\lambda_{c}+P^{\mathrm{D}} \sum_{j=1}^{J_{k \mid k-1}} w_{k \mid k-1}^{j} \mathcal{N}\left(z ; h\left(m_{k \mid k-1}^{j}\right), S_{k}^{j}\right)}
\end{aligned}
$$

and $J_{k \mid k-1}$ is the number of GM components in $v_{k \mid k-1}(x)$. The updated posterior and predicted likelihood are estimated using the EKF [17]

$$
\begin{aligned}
S_{k}^{i} & =H_{x}\left(m_{k \mid k-1}^{i}\right) P_{k \mid k-1}^{i}\left[H_{x}\left(m_{k \mid k-1}^{i}\right)\right]^{\top}+\Sigma, \\
K_{k}^{i} & =P_{k \mid k-1}^{i}\left[H_{x}\left(m_{k \mid k-1}^{i}\right)\right]^{\top}\left[S_{k}^{i}\right]^{-1}, \\
m_{k \mid k}^{i} & =m_{k \mid k-1}^{i}+K_{k}^{i}\left(z_{k}-h\left(m_{k \mid k-1}^{i}\right)\right), \\
P_{k \mid k}^{i} & =P_{k \mid k-1}^{i}-K_{k}^{i} S_{k}^{i}\left[K_{k}^{i}\right]^{\top},
\end{aligned}
$$

${ }^{2}$ Measurement $z_{k}^{j}$ is not associated to any existing landmark if $d^{j, i}>$ $T_{G} \forall i$, where $d^{j, i}=\left(z_{k}^{j}-h\left(m_{k \mid k-1}^{i}\right)\right)^{\top} S_{k}^{i}\left(z_{k}^{j}-h\left(m_{k \mid k-1}^{i}\right)\right), h(\cdot)$ the measurement model, $S_{k}^{i}$ the innovation covariance and $T_{G}$ is the gating threshold 
where the measurement model is

$$
h\left(m_{k \mid k-1}^{i}\right)=\left[\begin{array}{c}
\sqrt{\left(\hat{x}-x^{u e}\right)^{2}+\left(\hat{y}-y^{u e}\right)^{2}} \\
\arctan \left(\frac{\hat{y}-y^{u e}}{\hat{x}-x^{u e}}\right)
\end{array}\right]
$$

and the Jacobian of $h(\cdot)$ has elements $\left\{H_{x}\left(m_{k \mid k-1}^{i}\right)\right\}_{l, m}=$ $\partial h_{l}(x) /\left.\partial x_{m}\right|_{x=m_{k \mid k-1}^{i}}$, given by

$$
H_{x}\left(m_{k \mid k-1}^{i}\right)=\left[\begin{array}{cc}
\frac{\left(\hat{x}-x^{u e}\right)}{\sqrt{\left(\hat{x}-x^{u e}\right)^{2}+\left(\hat{y}-y^{u e}\right)^{2}}} & \frac{\left(\hat{y}-y^{u e}\right)}{\sqrt{\left(\hat{x}-x^{u e}\right)^{2}+\left(\hat{y}-y^{u e}\right)^{2}}} \\
-\frac{\hat{y}-y^{u e}}{\left(\hat{x}-x^{u e}\right)^{2}+\left(\hat{y}-y^{u e}\right)^{2}} & \frac{\hat{x}-x^{u e}}{\left(\hat{x}-x^{u e}\right)^{2}+\left(\hat{y}-y^{u e}\right)^{2}}
\end{array}\right] .
$$

\section{NumERICAL EVAluATION AND ANALYSIS}

In this section, we evaluate the performance of the proposed EK-PHD, first with a realistic ray-tracing-based channel model and then with a simplified measurement model, to perform a quantitative analysis.

\section{A. Evaluation with Realistic Ray-tracing Data}

We use a realistic ray-tracing simulations in a real-life indoor office environment at the Tampere University, Finland. The raytracing simulations include a user track of 31 measurement instances with $1 \mathrm{~m}$ intervals in an approximately $2 \mathrm{~m}$ wide corridor. The considered indoor layout, which is relatively challenging due to its diverse and irregular wall structures, is illustrated together with the mapping results in Fig. 2.

1) Data Generation: The ray-tracing simulations are performed by using the Wireless Insite $\AA$ software [18], where the maximum number of propagation paths per location is configured to 25 with at most 6 reflections and 1 diffraction. Moreover, the walls, floor and ceiling are configured with appropriate RF-specific materials to reflect the radio link behavior in real-life environment. Based on the 3D propagation path data provided by the ray-tracing simulator, including path coefficients, path delays and related path angular data, the received samples are generated according to (1).

At each measurement location, the UE performs an ana$\log$ beamsweeping procedure, consisting of transmissions of 14 quadrature phase shift keying (QPSK) modulated OFDM symbols into the considered beam directions in azimuth plane as $\left\{-180^{\circ},-178^{\circ}, \ldots, 178^{\circ}\right\}$. In order to achieve unambiguous angle measurements for the full $360^{\circ}$ sweeping range, the transmit and receive arrays are determined as a 16 element Uniform Circular Array (UCA) with radius of $1.27 \mathrm{~cm}$. Each array element has an omnidirectional azimuth radiation pattern and a 3GPP-specified vertical radiation pattern using a $16^{\circ}$ $3 \mathrm{~dB}$-beamwidth as defined in [19]. The used OFDM waveform follows the available 5G NR numerology at $30 \mathrm{GHz}$ carrier frequency with subcarrier spacing of $120 \mathrm{kHz}$. Furthermore, in order to optimize the ranging resolution, we consider the maximum resource allocation for the used frequency range, namely the $400 \mathrm{MHz}$ bandwidth, which contains 264 resource blocks, and thus 3168 active subcarriers. Based on the above, the rangeangle measurements for the EK-PHD filter are obtained from the received IQ samples during the beamsweeping procedure as described in [14]. However, we note that for the proposed EKPHD filter it is irrelevant in which specific manner the rangeangle measurements are obtained.

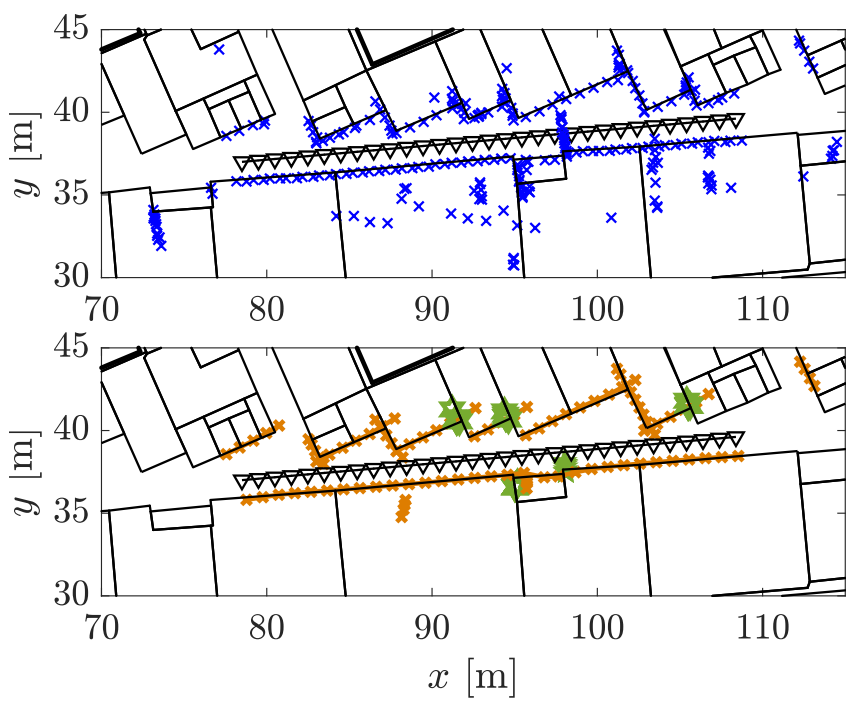

Fig. 2. Dynamic mapping performance using ray-tracing data. On top, the UE location illustrated with black triangles and ray-tracing measurements using blue crosses. On the bottom, the estimated reflectors are mapped with orange lines/crosses and the estimated scatterers using green hexagrams.

2) Data Analysis: The proposed EK-PHD filter for the dynamic mapping is parameterized as follows. The measurement noise covariance is set to $\Sigma=\operatorname{diag}\left(\left[0.1 \mathrm{~m}^{2}, 0.01 \mathrm{rad}^{2}\right]\right)$ and clutter intensity to $\lambda_{c}=10^{-3}$. In addition, detection, survival and birth probabilities are determined as $P^{\mathrm{D}}=0.9$, $P^{\mathrm{S}}=0.9$ and $P^{\mathrm{B}}=10^{-3}$, respectively. In order to decrease the computational complexity, pruning, merging, capping and ellipsoidal gating are utilized, and readers are referred to [15] for further details. The corresponding parameters are given as: pruning threshold $\left(10^{-3}\right)$, merging threshold 50, capping number 50 and gating threshold 4.6052, which is calculated using the chi-square distribution with two degrees of freedom.

In Fig. 2, the range-angle measurements converted to $2 \mathrm{D}$ Euclidean coordinates and the dynamic mapping results of the proposed EK-PHD filter are illustrated for the ray-tracing based simulation data. In the illustrated results, the following post processing steps are carried out to remove redundant and false landmarks. First, trajectories that are three samples or shorter are removed. Second, for each birth only the landmark that results in the highest average weight is considered. As seen in Fig. 2, specular reflections are dominant in the considered environment, and scatterers are evaluated only in five specific locations with nearby corners. For the most part, the EK-PHD can accurately estimate the landmark locations; however, two exceptions occur. The first case is a false detection, created by a triple reflection, which is observed as an artifact below the lower side wall of the corridor around the $x-y$-coordinate $(88 \mathrm{~m}, 35 \mathrm{~m})$. The second case is a false classification where a wall is mistakenly classified as a scatterer. This scenario is encountered three times on the upper side of the corridor, as seen in Fig. 2. In each case, the UE movement is close to perpendicular to the wall and it is not apparent what the landmark type is. The EK-PHD correctly tracks both landmarks 

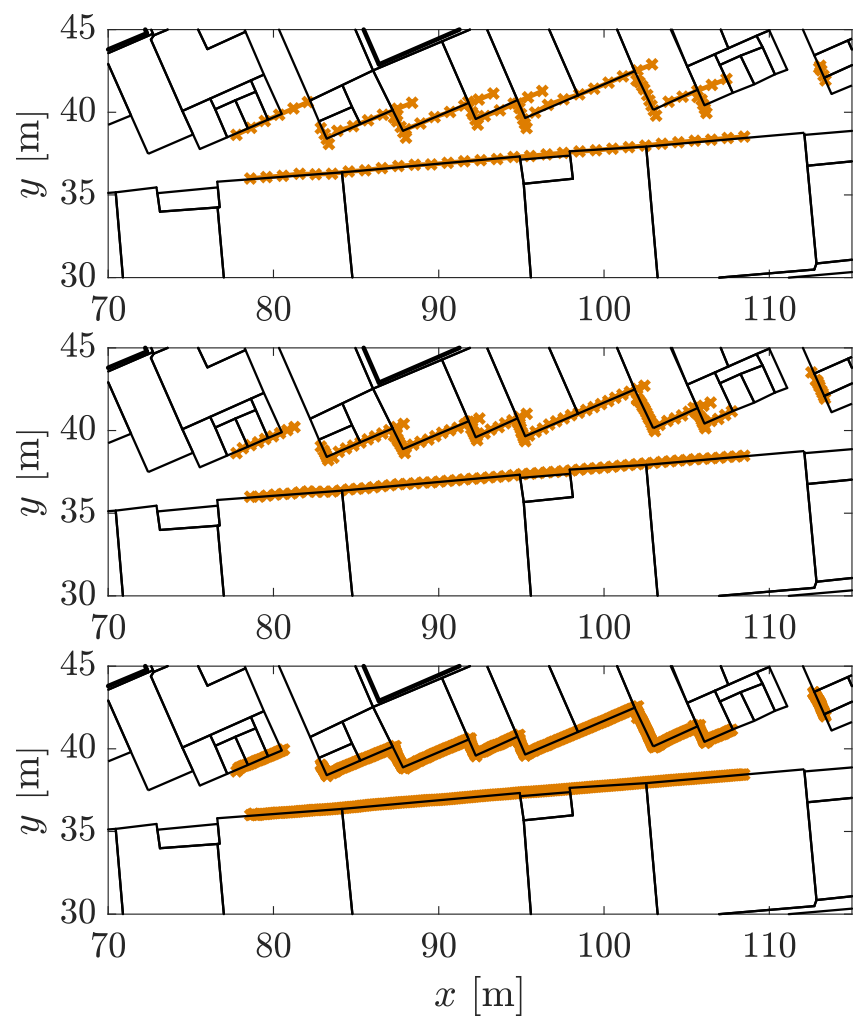

Fig. 3. Dynamic mapping performance using single bounce reflection data and with different sampling intervals: $100 \mathrm{~cm}$ (top), $50 \mathrm{~cm}$ (middle) and $10 \mathrm{~cm}$ (bottom).

but in post processing, the scattering landmark has a higher average weight resulting to a wrong decision.

\section{B. Evaluation with Simplified Measurement Model}

To gain deeper insight into the performance of the proposed EK-PHD filter, we further evaluate a simplified measurement model, where the filter performance can be analyzed with welldefined statistical range-angle measurements. It is noted that in realistic deployments, such as with ray-tracing, it is often difficult to observe the root cause of certain mapping errors, which can occur, e.g., due to multibounce propagation. Thus, with the simplified measurement model, we are able to remove the effect of the unknown factors and focus on the characteristic of the proposed EK-PHD filter.

1) Data Generation: At each UE position in the considered indoor layout, range-angle measurements for the single bounce reflections are generated and corrupted by additive Gaussian noise with covariance $\Sigma=\operatorname{diag}\left(\left[0.01 \mathrm{~m}^{2}, 0.001 \mathrm{rad}^{2}\right]\right)$. Besides the used $1 \mathrm{~m}$ measurement sample interval with the ray-tracing data, we consider also $50 \mathrm{~cm}$ and $10 \mathrm{~cm}$ sample intervals with the simplified data for further performance analysis.

2) Data Analysis: The mapping results for the proposed EKPHD filter, using exactly the same filter parameterization as earlier with the ray-tracing data, is illustrated in Fig. 3 for three different measurement sampling intervals. Since in this setup the range-angle measurements $z_{k}^{j}=\left[\begin{array}{ll}d_{k}^{j} & \theta_{k}^{j}\end{array}\right]^{\top}$ originate from

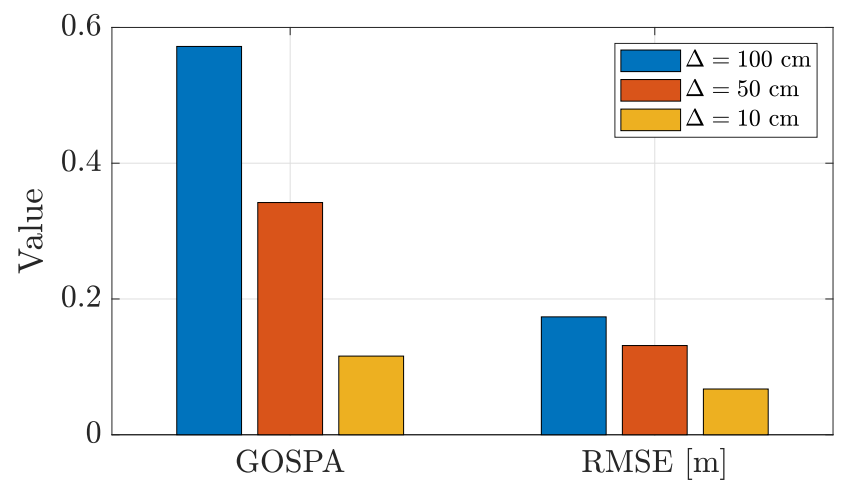

Fig. 4. GOSPA and RMSE with different sampling intervals.

a well-defined Gaussian distribution, they are not separately illustrated, as earlier with the ray-tracing data. The mapping results with the ray-tracing and single bounce reflection data closely resemble one another when the sampling interval is the same (i.e., $1 \mathrm{~m}$ ). In both simulation scenarios, the wall extensions are overestimated since the track can survive for one or two samples even though the wall is not detected. The filter would terminate the tracks sooner by considering smaller values for the detection probability $P^{\mathrm{D}}$ and survival probability $P^{\mathrm{S}}$. However, at the same time, some tracks could be terminated prematurely. Another option is to use a smaller sampling interval as shown in Fig. 3, where it is shown that the wall overshooting is considerably reduced by using $50 \mathrm{~cm}$ and $10 \mathrm{~cm}$ measurement sampling intervals.

For numerical evaluations, the root mean square error (RMSE) is used to measure the accuracy of the detected landmark estimates, and the mapping accuracy is assessed using the mean of the generalized optimal subpattern assignment (GOSPA) [20], which in addition to the landmark estimation accuracy, takes into account the effect of misdetected and falsely detected landmarks. For the RMSE and GOSPA, the landmark estimation accuracy is defined over different measurement samples according to Euclidean distance between the estimated sample-wise wall coordinates and the true incidence points of the corresponding single-bounce reflections. Overall, 100 Monte Carlo simulations are performed and the results are obtained by averaging over the different simulation trials. The results are summarized in Fig. 4, where the GOSPA and RMSE are shown for the three considered measurement sampling intervals. Besides observing the evident performance improvement while reducing the sampling interval, it is shown that the GOSPA metric clearly suffers more when the sampling interval is increased. This is because in the considered indoor scenario the shortest walls are just at meter level in length, and therefore, with comparable measurement sampling intervals the susceptibility to misdetections and false detections accumulates drastically as only a few samples can be obtained from the shortest walls.

In order to illustrate the time-evolution of the considered evaluation metrics, the above discussed GOSPA and RMSE are 

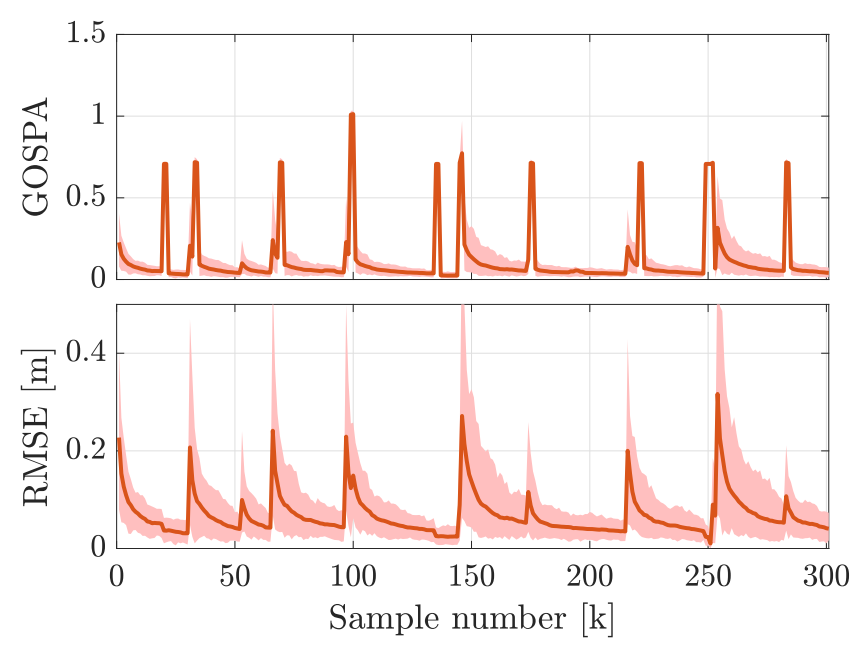

Fig. 5. GOSPA and RMSE as a function of sample number using $10 \mathrm{~cm}$ sampling interval. The red line depicts the mean and the shaded area illustrates the $95 \%$ confidence interval.

illustrated in Fig. 5 as a function of sample number. Regarding the GOSPA metric, the shown peaks correspond to situations when a wall begins or ends, in which cases the metric penalizes (one meter penalty) for misdetections and false detections. Correspondingly, the RMSE is higher whenever a new track is initialized and then by accumulating more measurements, the estimate gradually improves decreasing the RMSE.

\section{Conclusions}

In this paper, we proposed and derived a EK-PHD filter for dynamic environment mapping with UE-centric mobile radars. Based on realistic indoor channel simulation with ray-tracing, we demonstrated that the proposed approach is able to produce accurate mapping of landmarks, including reflective walls and fixed scattering objects. Furthermore, for numerical evaluations we considered a simplified measurement model, where rangeangle measurements are originated only from single bounce specular reflections. By comparing the performance of the EKPHD filter between the considered evaluation metrics, namely the GOSPA and RMSE, we showed that decreasing the measurement sampling interval is especially beneficial to GOSPA, where the target misdetection and false detection, occuring often in the beginning and end part of a wall, are separately penalized.

Regardless of the presented promising initial results, there are certain development aspects to be considered in further research. First, it is desired to improve handling of the birth and death process of wall objects for enabling accurate mapping without decreasing the sampling interval. Second, it is important to mitigate the effect of multi-bounce propagation in order to remove unwanted mapping artifacts. Besides developing the proposed EK-PHD solution, an extension to 3D mapping as well as designing of efficient preprocessing and postprocessing algorithms for the mapping process, are also of great interest. Nonetheless, already as such, the proposed EK-PHD filter is able to provide high-quality mapping results with attractive computational efficiency, and thus, it can be considered a promising candidate for various sensing applications in $5 \mathrm{G}$ and upcoming $6 \mathrm{G}$ technologies.

\section{REFERENCES}

[1] C. De Lima et al., "Convergent Communication, Sensing and Localization in 6G Systems: An Overview of Technologies, Opportunities and Challenges," IEEE Access, vol. 9, pp. 26902-26925, 2021.

[2] F. Liu, C. Masouros, A. P. Petropulu, H. Griffiths, and L. Hanzo, "Joint Radar and Communication Design: Applications, State-of-the-Art, and the Road Ahead," IEEE Trans. on Commun., vol. 68, no. 6, pp. 3834-3862, 2020.

[3] T. Wild, V. Braun, and H. Viswanathan, "Joint Design of Communication and Sensing for Beyond 5G and 6G Systems," IEEE Access, vol. 9, pp. 30 845-30 857, 2021.

[4] C. Baquero Barneto et al., "Full-Duplex OFDM Radar With LTE and 5G NR Waveforms: Challenges, Solutions, and Measurements," IEEE Trans. Microw. Theory Tech., vol. 67, no. 10, pp. 4042-4054, 2019.

[5] A. Guerra, F. Guidi, D. Dardari, A. Clemente, and R. D'Errico, “A Millimeter-Wave Indoor Backscattering Channel Model for Environment Mapping," IEEE Trans. Antennas Propag., vol. 65, no. 9, pp. 4935-4940, 2017.

[6] F. Guidi, A. Guerra, and D. Dardari, "Personal Mobile Radars with Millimeter-Wave Massive Arrays for Indoor Mapping," IEEE Trans. Mobile Comput., vol. 15, no. 6, pp. 1471-1484, 2016.

[7] F. Guidi et al., "Indoor Environment-Adaptive Mapping With Beamsteering Massive Arrays," IEEE Trans. Veh. Technol., vol. 67, no. 10, pp. $10139-10143,2018$.

[8] C. Baquero Barneto et al., "Radio-based Sensing and Indoor Mapping with Millimeter-Wave 5G NR Signals," in 2020 Int. Conf. Localization and GNSS, 2020, pp. 1-5.

[9] E. Leitinger et al., "A Belief Propagation Algorithm for Multipath-Based SLAM," IEEE Trans. Wireless Commun., vol. 18, no. 12, pp. 5613-5629, 2019.

[10] H. Kim et al., "5G mmWave Cooperative Positioning and Mapping Using Multi-Model PHD Filter and Map Fusion," IEEE Trans. Wireless Commun., vol. 19, no. 6, pp. 3782-3795, 2020.

[11] C. Gentner et al., "Multipath Assisted Positioning with Simultaneous Localization and Mapping," IEEE Trans. Wireless Commun., vol. 15, no. 9, pp. 6104-6117, 2016.

[12] M. Adams, B.-N. Vo, R. Mahler, and J. Mullane, "SLAM Gets a PHD: New Concepts in Map Estimation," IEEE Robot. Automat. Mag., vol. 21, no. 2, pp. 26-37, 2014.

[13] J. H. Brady and A. M. Sayeed, "Wideband communication with highdimensional arrays: New results and transceiver architectures," in 2015 IEEE Int. Conf. Commun. Workshop, 2015, pp. 1042-1047.

[14] C. B. Barneto et al., "Radio-based Sensing and Environment Mapping in Millimeter-Wave 5G and Beyond Networks," 2021. [Online]. Available: https://arxiv.org/abs/2102.11593

[15] B.-N. Vo and W.-K. Ma, "The Gaussian Mixture Probability Hypothesis Density Filter," IEEE Trans. Signal Process., vol. 54, no. 11, pp. 40914104, 2006.

[16] R. Mahler, "Multi-target Bayes filtering via first-order multi-target moments," IEEE Trans. AES, vol. 39, no. 4, pp. 1152-1178, 2003.

[17] S. Särkkä, Bayesian Filtering and Smoothing, ser. Institute of Mathematical Statistics Textbooks. Cambridge University Press, 2013.

[18] Remcom. Wireless InSite - 3D Wireless Prediction Software. Accessed: Jan 27, 2021). [Online]. Available: https://www.remcom.com/wirelessinsite-em-propagation-software

[19] 3GPP TR 38.901, "Study on channel model for frequencies from 0.5 to $100 \mathrm{GHz}, "$ 2020. [Online]. Available: http://www.3gpp.org/dynareport/38901.htm

[20] A. S. Rahmathullah, A. F. García-Fernández, and L. Svensson, "Generalized optimal sub-pattern assignment metric," in 20th Int. Conf. Inform. Fusion, 2017, pp. 1-8. 